\title{
Hydrogen Bond-Induced Rigid Oligoanthranilamide Ribbons That Are Planar and Straight
}

\author{
Zong-Quan Wu, Xi-Kui Jiang, Shi-Zheng Zhu, Zhan-Ting Li* \\ Shanghai Institute of Organic Chemistry, Chinese Academy of Sciences, \\ 354 Fenglin Lu, Shanghai 200032, China
}

\section{Supporting Information}

Experiemtnal Procedures $\quad$ S2

Packing Diagram of $2 \quad$ S11

Packing Diagram of $3 \quad$ S12 


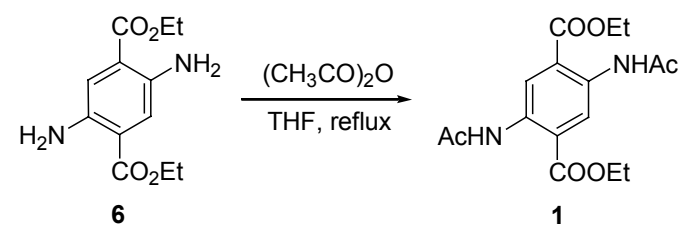

Compound 1. To a stirred solution of compound $6(0.24 \mathrm{~g}$, $)$ and triethylamine $(0.50 \mathrm{~mL})$ in THF $(25 \mathrm{~mL})$ was added dropwise acetic anhydride $(2.00 \mathrm{~mL})$ at room temperature. The mixture was then refluxed until 6 was consumed (about 5 hours). The solvent was removed under reduced pressure to give a residue, which was thoroughly washed with water. The resulting crude product was recrystallized from ethanol to afford $\mathbf{1}(0.22 \mathrm{~g}, 70 \%)$ as a pale green solid. ${ }^{1} \mathrm{H}$ NMR $\left(\mathrm{CDCl}_{3}\right) \delta: 10.90(\mathrm{~s}, 2 \mathrm{H}), 9.38(\mathrm{~s}, 2 \mathrm{H}), 4.42\left(\mathrm{q}, \mathrm{J}_{1}=7.1 \mathrm{~Hz}, \mathrm{~J}_{2}=14.2\right.$ Hz, 4 H), 2.22 (s, 6 H), 1.44 (t, J = 7.1 Hz, 6 H). MS (EI): m/z $337\left(\mathrm{M}^{+}+\mathrm{H}\right)$.
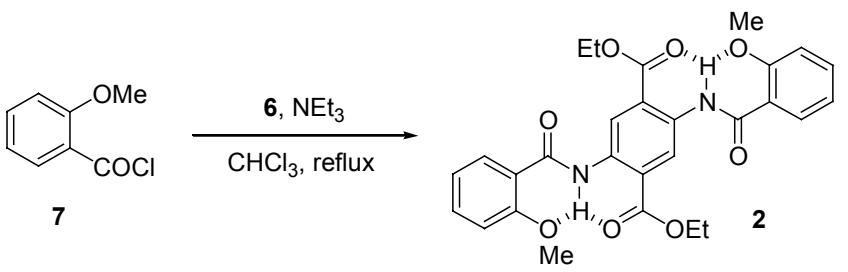

Compound 2. To a stirred solution of $6(0.24 \mathrm{~g}, 0.95 \mathrm{mmol})$ and triethylamine $(0.50 \mathrm{~mL})$ in chloroform $(30 \mathrm{~mL})$ was added a solution of $7(0.42 \mathrm{~g}, 2.50 \mathrm{mmol})$ in chloroform $(5 \mathrm{~mL})$ at room temperature. The solution was then heated under reflux for $2 \mathrm{hrs}$. Upon cooling to room temperature, the solution was washed with aqueous $\mathrm{K}_{2} \mathrm{CO}_{3}$ solution $(1 \mathrm{~N}, 10 \mathrm{~mL} \times 2)$, water, brine, and dried over $\mathrm{MgSO}_{4}$. After removal of the solvent under reduced pressure, the resulting residue was subjected to column chromatography $\left(\mathrm{CH}_{2} \mathrm{Cl}_{2} / \mathrm{MeOH}\right.$ 250:1). Compound $2(0.25 \mathrm{~g}, 52 \%)$ was obtained as a greenish solid. M.p. $218-220{ }^{\circ} \mathrm{C} .{ }^{1} \mathrm{H}$ NMR $\left(\mathrm{CDCl}_{3}\right) \delta: 12.17(\mathrm{~s}, 2 \mathrm{H}), 9.64(\mathrm{~s}, 2 \mathrm{H}), 8.24(\mathrm{~d}, \mathrm{~J}=7.7 \mathrm{~Hz}, 2 \mathrm{H}), 7.52-7.46(\mathrm{~m}, 2 \mathrm{H}), 7.05(\mathrm{~m}$, $4 \mathrm{H}), 4.45\left(\mathrm{q}, \mathrm{J}_{1}=1.5 \mathrm{~Hz}, \mathrm{~J}_{2}=6.6 \mathrm{~Hz}\right), 4.10(\mathrm{~s}, 6 \mathrm{H}), 1.56(\mathrm{t}, \mathrm{J}=7.2 \mathrm{~Hz}, 6 \mathrm{H}) . \mathrm{MS}(\mathrm{EI}): \mathrm{m} / z$ $520\left(\mathrm{M}^{+}\right)$. Anal. Calcd. for $\mathrm{C}_{28} \mathrm{H}_{28} \mathrm{~N}_{2} \mathrm{O}_{8}: \mathrm{C}, 64.64 ; \mathrm{H}, 5.42 ; \mathrm{N}, 5.38$. Found: $\mathrm{C}, 64.52 ; \mathrm{H}, 5.25$; N, 5.26. 

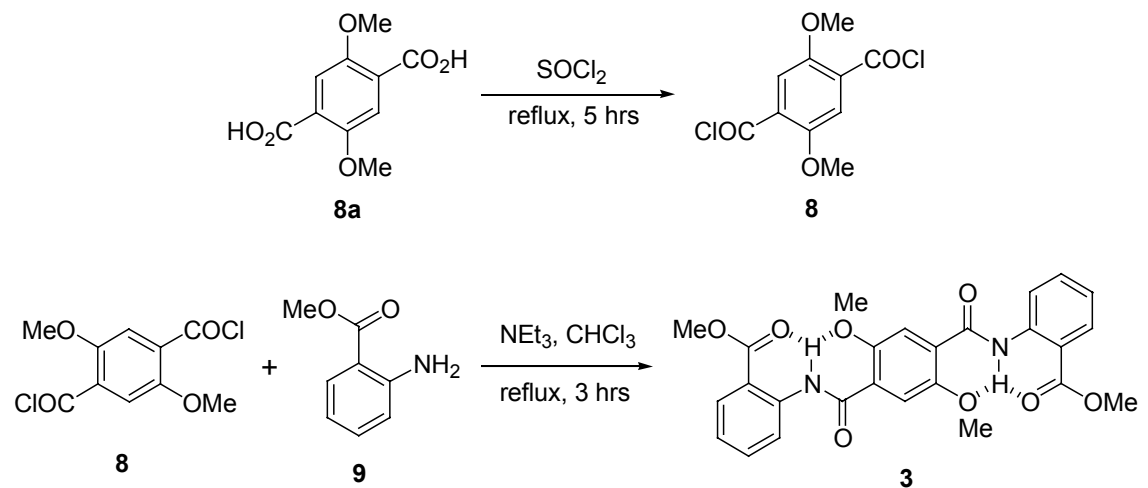

Compound 3. Compound 8a $(0.19 \mathrm{~g}, 0.88 \mathrm{mmol})$ was added to thionyl chloride $(5 \mathrm{~mL})$. The suspension was heated under reflux for $5 \mathrm{hrs}$ and then concentrated under reduced pressure to afford 8 which was used for the next step without further purification. Compound 9 ( $0.28 \mathrm{~g}$, $1.80 \mathrm{mmol})$ was dissolved in chloroform $(20 \mathrm{~mL})$ and triethylamine $(0.50 \mathrm{~mL})$ at room temperature and then a solution of compound 8, prepared as described above, in chloroform $(10 \mathrm{~mL})$ was added dropwise with stirring. The solution was heated under reflux for $3 \mathrm{hrs}$ and then cooled to room temperature. The precipitate formed was filtered and washed with petroleum ether $\left(30-60{ }^{\circ} \mathrm{C}\right)$. After recrystallization from chloroform, compound $\mathbf{3}$ was obtained as white solid (0.28 g, 62\%). M.p. $255-257{ }^{\circ} \mathrm{C} .{ }^{1} \mathrm{H}$ NMR $\left(\mathrm{CDCl}_{3}\right) \delta: 12.37$ (s, $\left.2 \mathrm{H}\right)$, 8.92 (s, 2 H), 8.07 (d, J = 7.8 Hz, 2 H), 7.95 (d, 2 H), 7.60 (m, 2 H), 7.15 (m, 2 H), 4.15 (s, 6 H), 3.95 (s, $6 \mathrm{H})$. MS (EI): $m / z 492\left(\mathrm{M}^{+}\right)$. Anal. Calcd. for $\mathrm{C}_{26} \mathrm{H}_{24} \mathrm{O}_{8} \mathrm{~N}_{2}: \mathrm{C}, 63.41 ; \mathrm{H}, 4.87 ; \mathrm{N}$, 5.69. Found: C, 63.08; H, 4.85; N, 5.60.
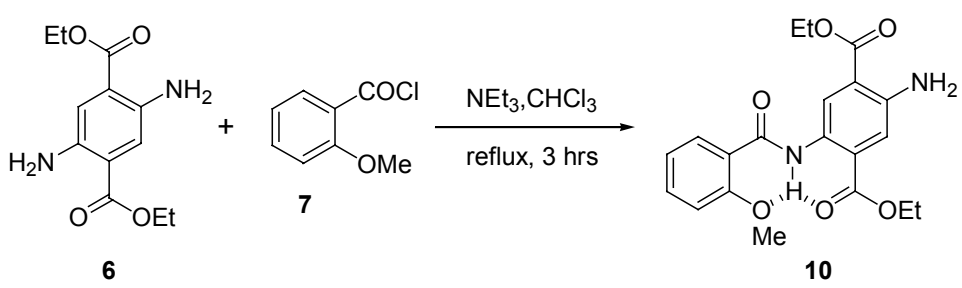

Compound 10. This compound was prepared as a pale yellow solid in $70 \%$ yield from the reaction of 6 (2.0 equiv.) and 7 (1.0 equiv.) in chloroform according to the precedure described for preparation of 3. M.p. $114-116{ }^{\circ} \mathrm{C} .{ }^{1} \mathrm{H} \mathrm{NMR}\left(\mathrm{CDCl}_{3}\right) \delta: 11.70(\mathrm{~s}, 1 \mathrm{H}), 9.35(\mathrm{~s}, 1$ H), $8.25(\mathrm{~d}, \mathrm{~J}=7.7 \mathrm{~Hz}, 1 \mathrm{H}), 7.49(\mathrm{~m}, 1 \mathrm{H}), 7.35(\mathrm{~s}, 1 \mathrm{H}), 7.03(\mathrm{~m}, 2 \mathrm{H}), 4.38(\mathrm{~m}, 4 \mathrm{H}), 4.07$ 
(s, $3 \mathrm{H}), 4.13$ (m, $6 \mathrm{H})$. MS (EI): m/x $386\left(\mathrm{M}^{+}\right)$. Anal .Calcd. for $\mathrm{C}_{20} \mathrm{H}_{22} \mathrm{O}_{6} \mathrm{~N}_{2}$ : C, 62.18; H, 5.70; N, 7.25. Found. C, 62.22; H, 5.73; N, 7.28.<smiles>COc1cc(C(=O)Cl)c(OC)cc1C(=O)[O-]</smiles>

8

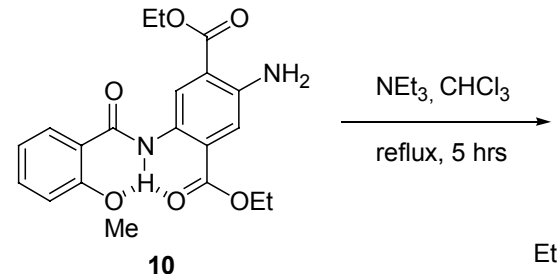

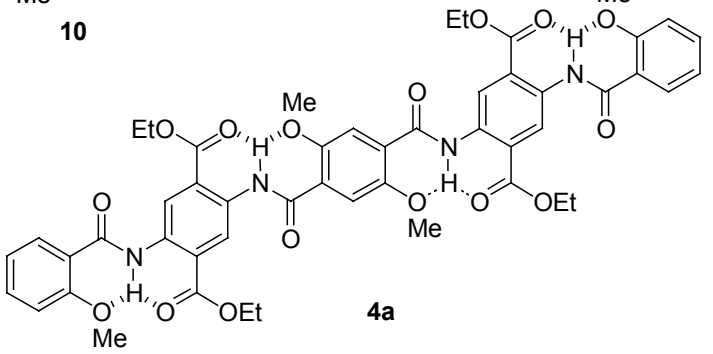

Compound 4a. This compound was prepared as a yellow solid in 52\% yield from the reaction of 8 and $\mathbf{1 0}$ according to the procedure described for preparation of 3. M.p. $200{ }^{\circ} \mathrm{C} .{ }^{1} \mathrm{H}$ NMR $\left(\mathrm{CDCl}_{3}\right) \delta: 12.34(\mathrm{~s}, 2 \mathrm{H}), 12.18(\mathrm{~s}, 2 \mathrm{H}), 9.66(\mathrm{~s}, 2 \mathrm{H}), 9.64(\mathrm{~s}, 2 \mathrm{H}), 8.24\left(\mathrm{q}, \mathrm{J}_{1}=1.5 \mathrm{~Hz}, \mathrm{~J}_{2}=\right.$ $7.5 \mathrm{~Hz}, 2 \mathrm{H}), 7.99$ (s, $2 \mathrm{H}), 7.50-7.47(\mathrm{~m}, 2 \mathrm{H}), 7.13-7.04(\mathrm{~m}, 4 \mathrm{H}), 4.47$ (q, J $\mathrm{J}_{1}=6.9 \mathrm{~Hz}, \mathrm{~J}_{2}=$ $14.1 \mathrm{~Hz}, 8 \mathrm{H}), 4.15(\mathrm{~s}, 6 \mathrm{H}), 4.13(\mathrm{~s}, 6 \mathrm{H}), 1.47\left(\mathrm{t}, \mathrm{J}_{1}=7.2 \mathrm{~Hz}, \mathrm{~J}_{2}=14.1 \mathrm{~Hz}, 12 \mathrm{H}\right)$. MS (MALDI): $m / z 985.4(\mathrm{M}+\mathrm{Na})^{+}$. Anal. Calcd. for $\mathrm{C}_{50} \mathrm{H}_{50} \mathrm{O}_{16} \mathrm{~N}_{4} \cdot 0.5 \mathrm{H}_{2} \mathrm{O}: \mathrm{C}, 61.79 ; \mathrm{H}, 5.15 ; \mathrm{N}$, 5.76. Found: C, 61.78; H, 5.49, N, 5.58.

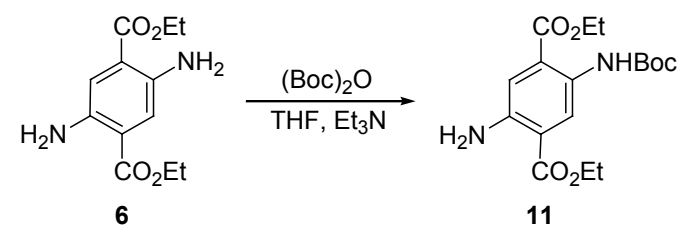

Compound 11. A solution of 6 (0.64 g, $2.56 \mathrm{mmol})$, di-t-butyl dicarbonate $(0.20 \mathrm{~g}, 0.97 \mathrm{mmol})$ and triethylamine $(0.50 \mathrm{~mL})$ in THF $(50 \mathrm{~mL})$ was stirred at room temperature for $12 \mathrm{hrs}$. The solvent was removed under reduced pressure and the resulting residue dissolved in AcOEt (80 $\mathrm{mL})$. The solution was washed with water $(15 \mathrm{~mL} \times 2)$, brine $(15 \mathrm{~mL})$, and dried over $\mathrm{MgSO}_{4}$. Upon removal of the solvent, the crude product was subject to column chromatography (petroleum ether/AcOEt 10:1) to give $\mathbf{1 1}$ as a yellow solid $(0.81 \mathrm{~g}, 90 \%)$. M.p. $116-118{ }^{\circ} \mathrm{C}$. 


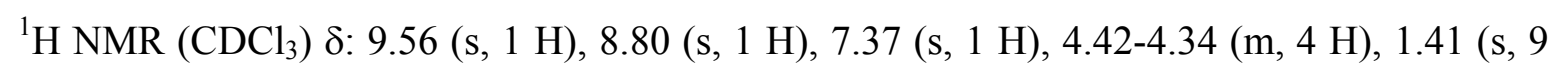
H). MS (EI): $m / z 352\left(\mathrm{M}^{+}\right)$. Anal. Calcd. for $\mathrm{C}_{17} \mathrm{H}_{24} \mathrm{O}_{6} \mathrm{~N}_{2}$ : C, 57.95; H, 6.82; N, 7.95. Found: C, 58.26; H, 6.91; N, 7.93 .

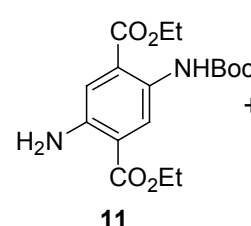

11

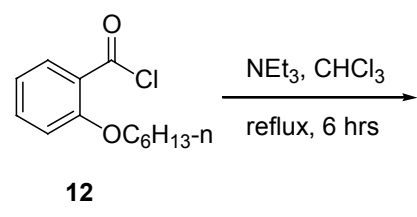

12

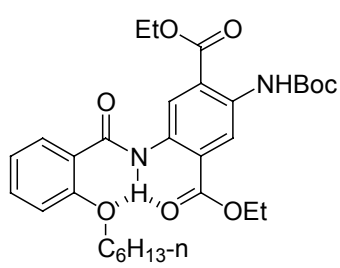

13

Compound 13. This compound was prepared as a yellow solid (98\%) from the reaction of $\mathbf{1 1}$ and 12 according to the condition for preparation of 4a. M.p. $118-120{ }^{\circ} \mathrm{C} .{ }^{1} \mathrm{H} \mathrm{NMR}\left(\mathrm{CDCl}_{3}\right) \delta$ : $11.73(\mathrm{~s}, 1 \mathrm{H}), 10.13(\mathrm{~s}, 1 \mathrm{H}), 9.46(\mathrm{~s}, 1 \mathrm{H}), 9.05(\mathrm{~s}, 1 \mathrm{H}), 8.17\left(\mathrm{~d} \mathrm{~d}, \mathrm{~J}_{1}=1.5 \mathrm{~Hz}, \mathrm{~J}_{2}=7.5 \mathrm{~Hz}, 1\right.$ H), $7.45(\mathrm{t}, \mathrm{J}=0.9 \mathrm{~Hz}, 1 \mathrm{H}), 7.04(\mathrm{~m}, 2 \mathrm{H}), 4.41(\mathrm{~m}, 4 \mathrm{H}), 4.23(\mathrm{t}, \mathrm{J}=6.6 \mathrm{~Hz}, 2 \mathrm{H}), 1.89-1.85$ (m, $2 \mathrm{H}), 1.46-1.24(\mathrm{~m}, 6 \mathrm{H}), 0.82(\mathrm{t}, \mathrm{J}=7.2 \mathrm{~Hz}, 3 \mathrm{H})$. MS (EI): $m / z 556\left(\mathrm{M}^{+}\right)$. Anal. Calcd. for $\mathrm{C}_{30} \mathrm{H}_{40} \mathrm{O}_{8} \mathrm{~N}_{2}$ : C, 64.75; H, 7.19; N, 5.04. Found: C, 64.92; H, 7.35; N, 4.85.
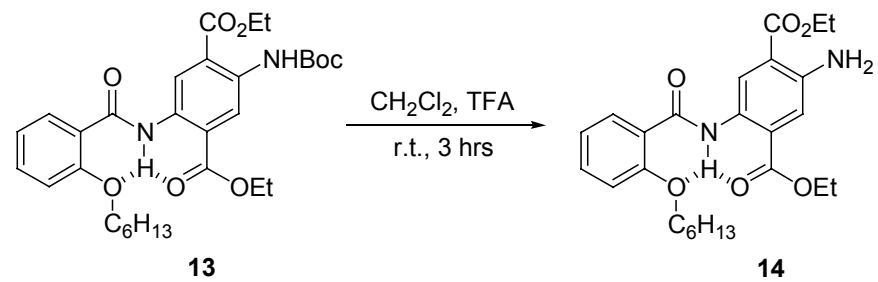

Compound 14. To a solution of $13(0.98 \mathrm{~g}, 1.81 \mathrm{mmol})$ in dichloromethane $(20 \mathrm{~mL})$ was added trifluoroacetic acid $(5 \mathrm{~mL})$. The solution was stirred at room temperature for $3 \mathrm{hrs}$ and then washed with water $(10 \mathrm{~mL} \times 3)$, brine $(10 \mathrm{~mL})$, and dried over $\mathrm{MgSO}_{4}$. After removal of the solvent, the residue was subjected to flash chromatography $\left(\mathrm{CHCl}_{3} / \mathrm{MeOH} 20: 1\right)$ to afford the title compound as a yellow solid (0.79 g, 96\%). M.p. 80-82 ${ }^{\circ} \mathrm{C} .{ }^{1} \mathrm{H}$ NMR $\left(\mathrm{CDCl}_{3}\right) \delta: 11.38$ (s, $1 \mathrm{H}), 9.20(\mathrm{~s}, 1 \mathrm{H}), 8.14\left(\mathrm{~d} \mathrm{~d}, \mathrm{~J}_{1}=7.5 \mathrm{~Hz}, \mathrm{~J}_{2}=1.5 \mathrm{~Hz}, 1 \mathrm{H}\right), 7.44-7.38$ (m, $\left.2 \mathrm{H}\right), 7.08-6.99$ $(\mathrm{m}, 2 \mathrm{H}), 4.36(\mathrm{t}, \mathrm{J}=6.9 \mathrm{~Hz}, 2 \mathrm{H}), 1.87$ (t, J = 3.9 Hz, $2 \mathrm{H}), 1.44-1.24(\mathrm{~m}, 6 \mathrm{H}), 0.80$ (t, J = $10.4 \mathrm{~Hz}, 3 \mathrm{H})$. MS (EI): $m / z 456\left(\mathrm{M}^{+}\right)$. Anal. Calcd. for $\mathrm{C}_{25} \mathrm{H}_{32} \mathrm{O}_{6} \mathrm{~N}_{2}: \mathrm{C}, 65.79 ; \mathrm{H}, 7.02 ; \mathrm{N}$, 6.14. Found: C, 65.86; H, 7.11; N, 5.83. 


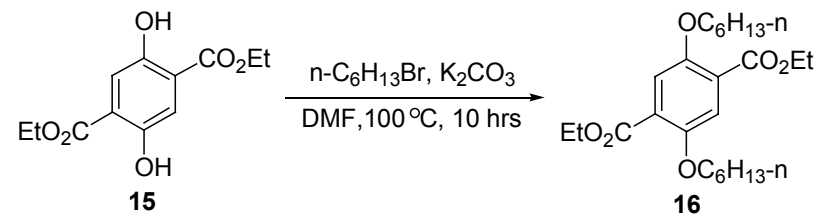

Compound 16. To a solution of compound 15 (2.10 g, $8.27 \mathrm{mmol})$ and 1-bromo-n-haxane $(3.30 \mathrm{~g}, 20.0 \mathrm{mmol})$ in $\mathrm{DMF}(50 \mathrm{~mL})$ was added $\mathrm{K}_{2} \mathrm{CO}_{3}(5.00 \mathrm{~g}, 38.0 \mathrm{mmol})$ at room temperature. The suspension was stirred at $90{ }^{\circ} \mathrm{C}$ for $12 \mathrm{hrs}$ and the solvent was removed under reduced pressure. The resulting residue was triburated with AcOEt $(80 \mathrm{~mL})$ and the solution was washed with water $(10 \mathrm{~mL} \times 2)$, brine $(10 \mathrm{~mL})$, and dried over $\mathrm{MgSO}_{4}$. The solvent was then distilled and the resulting solid was subjected to column chromatograph $\left(\mathrm{CH}_{2} \mathrm{Cl}_{2} /\right.$ petroleum ether 1:1) to afford compound 18 as a white solid (240 g, 69\%). M.p.

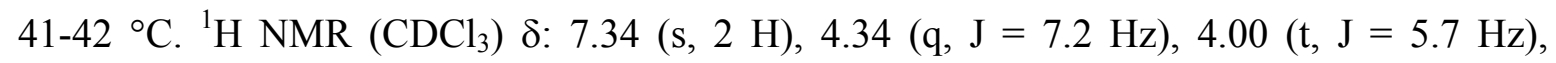
1.82-1.64 (m, 4 H), 1.5-1.26 (m, 8 H), 0.93-0.84 (m, 12 H). MS (EI): $m / z 422\left(\mathrm{M}^{+}\right)$. Anal. Calcd. for $\mathrm{C}_{24} \mathrm{H}_{38} \mathrm{O}_{6}$ : C, 68.24; H, 9.00. Found: C, 68.39; H, 9.20.

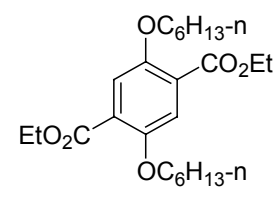

16

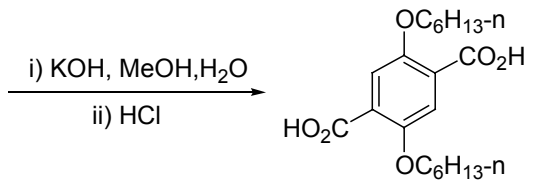

17

Compound 17. A solution of compound 16 (2.40 g, $5.71 \mathrm{mmol})$ and $\mathrm{KOH}(1.00 \mathrm{~g}, 17.2 \mathrm{mmol})$ in $\mathrm{MeOH}-\mathrm{H}_{2} \mathrm{O}(60 \mathrm{~mL}, 2: 1)$ was heated under reflux for $3 \mathrm{hrs}$. The solution was concentrated to $20 \mathrm{~mL}$ and hydrochloric acid $(2 \mathrm{~N})$ was added to $\mathrm{pH}=4$. The precipitate was filtered and washed with cold methanol to give compound 17 as a white solid (2.01 g, 96\%). M.p. 140-142 ${ }^{\circ} \mathrm{C} .{ }^{1} \mathrm{H}$ NMR (DMSO-d 6 ) $\delta: 12.80$ (s, $\left.2 \mathrm{H}\right), 7.20$ (s, $\left.2 \mathrm{H}\right), 3.94$ (s, $\left.4 \mathrm{H}\right), 1.62$ (d, J = $6.0 \mathrm{~Hz}, 4 \mathrm{H}), 1.38-1.26(\mathrm{~m}, 12 \mathrm{H}), 0.84(\mathrm{~s}, 6 \mathrm{H}) . \mathrm{MS}(\mathrm{EI}): \mathrm{m} / z 366\left(\mathrm{M}^{+}\right)$. Anal. Calcd. for $\mathrm{C}_{20} \mathrm{H}_{30} \mathrm{O}_{6}$ : C, 65.57; H, 8.12. Found: C, 65.52; H, 8.27. 


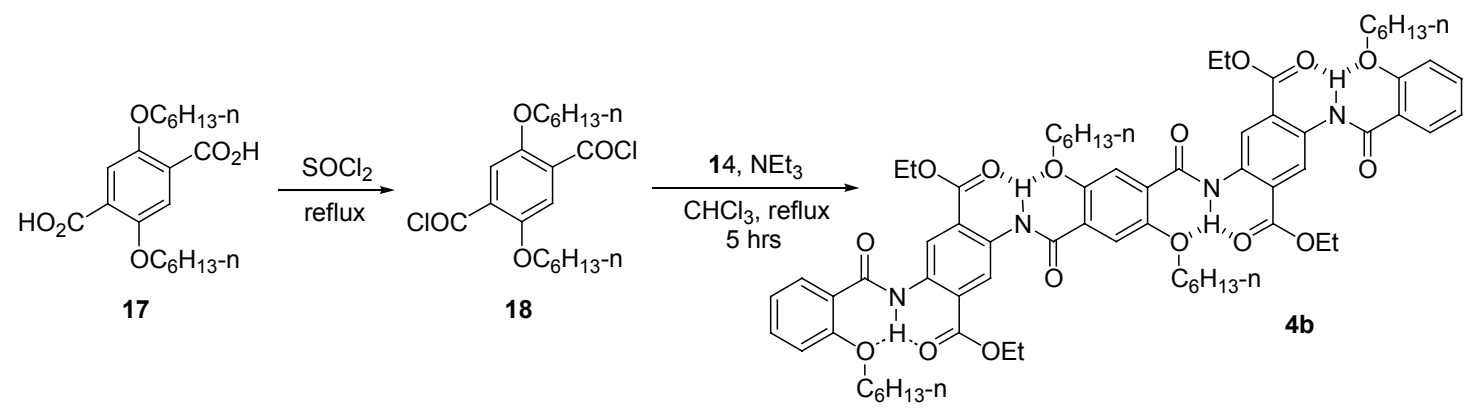

Compound $\mathbf{4 b}$. Compound 17 was first converted to 18 by refluxing in thionyl chloride for 5 hrs. The later reacted with $\mathbf{1 4}$, following the procedure described above to prepare $\mathbf{4 a}$, to give 4b as a greenish solid (65\%) after work-up and column chromatography (petroleum ether/AcOEt 7:1). M.p. 171-173 ${ }^{\circ} \mathrm{C} .{ }^{1} \mathrm{H}$ NMR $\left(\mathrm{CDCl}_{3}\right)$ $\delta: 11.92$ (s, $2 \mathrm{H}$ ), 9.53 (s, $\left.2 \mathrm{H}\right), 9.48$ (s, $2 \mathrm{H}), 8.12\left(\mathrm{~d} \mathrm{~d}, \mathrm{~J}_{1}=1.5 \mathrm{~Hz}, \mathrm{~J}_{2}=8.1 \mathrm{~Hz}, 2 \mathrm{H}\right), 7.85(\mathrm{~s}, 2 \mathrm{H}), 7.44(\mathrm{~m}, 2 \mathrm{H}), 7.04-7.01(\mathrm{~m}, 4 \mathrm{H})$, 4.46-4.39 (m, 8 H), 4.33-4.23 (m, 8 H), 1.94-1.84 (m, 8 H), 1.47-1.27 (m, 34 H), 0.88-0.82 (m, $12 \mathrm{H})$. MS (ESI): $m / z 1244.8\left(\mathrm{M}^{+}\right)$. Anal. Calcd. for $\mathrm{C}_{70} \mathrm{H}_{90} \mathrm{O}_{16} \mathrm{~N}_{4}: \mathrm{C}, 67.63 ; \mathrm{H}, 7.25 ; \mathrm{N}, 4.51$. Found: H, 67.94; H, 7.94; N, 4.02.

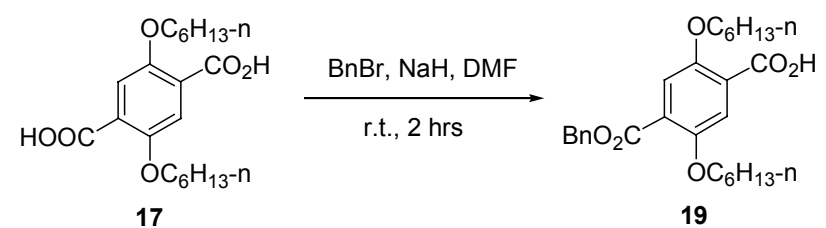

Compound 19. To a stirred solution of $17(0.87 \mathrm{~g}, 2.38 \mathrm{mmol})$ in dry DMF (50 mL) at about $0{ }^{\circ} \mathrm{C}$ was added $\mathrm{NaH}(60 \%, 0.10 \mathrm{~g})$. After 10 minutes, a solution of benzyl bromide $(0.41 \mathrm{~g}$, $2.40 \mathrm{mmol})$ in DMF $(2 \mathrm{~mL})$ was added. The solution was stirred at room temperature for 2 hours. Upon evaporation of the solvent under reduced pressure, the residue was triturated in dichloromethane $(60 \mathrm{~mL})$. The organic phase was washed with aqueous $\mathrm{HCl}$ solution $(1 \mathrm{~N}, 5$ $\mathrm{mL})$, water $(10 \mathrm{~mL} \times 3)$, brine $(10 \mathrm{~mL})$, and dried over $\mathrm{MgSO}_{4}$. After the solvent was removed, the crude product was chromatogaphed (petroleum ether/AcOEt 4:1) to afford 19 as a pale blue solid (0.68 g, 63\%). M.p. 66-68 ${ }^{\circ} \mathrm{C} .{ }^{1} \mathrm{H}$ NMR $\left(\mathrm{CDCl}_{3}\right) \delta: 11.24$ (s, $\left.1 \mathrm{H}\right), 7.76$ (s, 1 H), 7.47-7.38 (m, $5 \mathrm{H}), 5.38(\mathrm{~s}, 2 \mathrm{H}), 4.23(\mathrm{t}, \mathrm{J}=4.8 \mathrm{~Hz}, 2 \mathrm{H}), 4.03(\mathrm{t}, \mathrm{J}=3.6 \mathrm{~Hz}, 2 \mathrm{H})$, 1.94-1.84 (m, 4 H), 1.57-1.26 (m, 16 H), 0.91 (m, 6 H). MS (EI): m/z $456\left(\mathrm{M}^{+}\right)$. Anal.Calcd. 
for $\mathrm{C}_{27} \mathrm{H}_{36} \mathrm{O}_{6}: \mathrm{C}, 71.05 ; \mathrm{H}, 7.90$. Found: $\mathrm{C}, 71.07 ; \mathrm{H}, 8.04$.

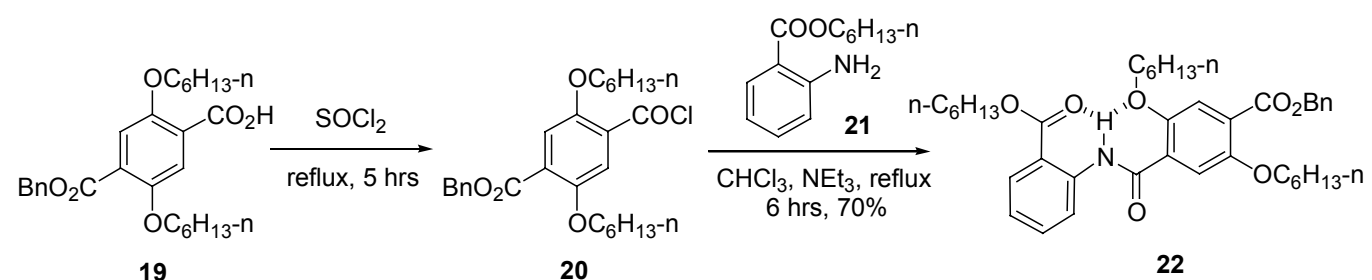

Compound 22. A suspension of $19(0.23 \mathrm{~g}, 0.50 \mathrm{mmol})$ in thionyl chloride $(12 \mathrm{~mL})$ was heated under reflux for 5 hours and then concentrated under reduced pressure to afford $\mathbf{2 0}$, which was dissolved in chloroform $(5 \mathrm{~mL})$ and used for the next step without further purification. To a solution of $21(0.11 \mathrm{~g}, 0.50 \mathrm{mmol})$ and $\mathrm{NEt}_{3}(0.2 \mathrm{~mL})$ in dry chloroform (20 $\mathrm{mL}$ ) was added the above solution of $\mathbf{2 0}$ at room temperautre. The solution was refluxed for 6 hours and then cooled to room temperature, washed with diluted $\mathrm{HCl}$ solution $(1 \mathrm{~N}, 10 \mathrm{~mL})$, saturated aqueous $\mathrm{NaHCO}_{3}$ solution $(10 \mathrm{~mL})$, water $(10 \mathrm{~mL})$, brine $(10 \mathrm{~mL})$, and dried over $\mathrm{MgSO}_{4}$. The solution was then concentrated and the resulting residue subjected to flash column chromatography $\left(\mathrm{CH}_{2} \mathrm{Cl}_{2}\right.$ /petroleum ether 5:3) to give $22(0.23 \mathrm{~g}, 70 \%)$ as a yellow oil. ${ }^{1} \mathrm{H}$ NMR $\left(\mathrm{CDCl}_{3}\right) \delta: 12.06(\mathrm{~s}, 1 \mathrm{H}), 8.01(\mathrm{~d}, \mathrm{~J}=7.2 \mathrm{~Hz}, 1 \mathrm{H}), 8.06(\mathrm{~d}, \mathrm{~J}=7.8 \mathrm{~Hz}, 1 \mathrm{H})$, $7.70(\mathrm{~s}, 1 \mathrm{H}), 7.61-7.55(\mathrm{~m}, 1 \mathrm{H})$, 7.49-7.36 (m, $6 \mathrm{H}), 7.24-7.12(\mathrm{~m}, 1 \mathrm{H}), 5.38(\mathrm{~s}, 2 \mathrm{H}), 4.29(\mathrm{t}$, $\mathrm{J}=6.6 \mathrm{~Hz}, 2 \mathrm{H}), 4.19(\mathrm{t}, \mathrm{J}=7.2 \mathrm{~Hz}, 2 \mathrm{H}), 4.04(\mathrm{t}, \mathrm{J}=6.6 \mathrm{~Hz}, 2 \mathrm{H}), 1.85-1.70(\mathrm{~m}, 6 \mathrm{H})$, 1.45-1.23 (m, $18 \mathrm{H}), 0.91-0.81(\mathrm{~m}, 9 \mathrm{H})$. MS (EI): $m / z 659\left(\mathrm{M}^{+}\right)$. Anal. Calcd. for $\mathrm{C}_{40} \mathrm{H}_{53} \mathrm{NO}_{7}: \mathrm{C}_{\text {, }}$ 72.84; H, 8.04; N, 2.12. Found: C, 72.95; H, 8.43; N, 2.06.

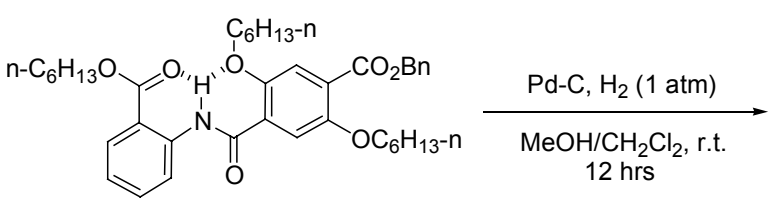

22

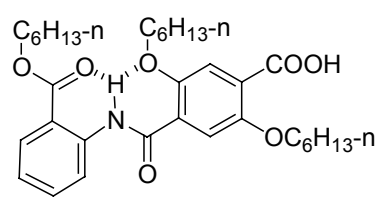

23

Compound 23. At room temperature, a solution of compound $22(0.23 \mathrm{~g}, 0.35 \mathrm{mmol})$ in $\mathrm{CH}_{2} \mathrm{Cl}_{2} / \mathrm{MeOH}(1: 1,30 \mathrm{~mL})$ was stirred under 1 atm of hydrogen in the presence of $10 \%$ $\mathrm{Pd} / \mathrm{C}(12 \mathrm{mg})$ for 5 hours. The catalyst was filtered and the solution was concentrated under reduced pressure. The crude product was then purified by column chromatography (petroleum 
ether/AcOEt 4:1) to afford $23(0.15 \mathrm{~g}, 75 \%)$ as a white solid. M.p. 53-55 ${ }^{\circ} \mathrm{C} .{ }^{1} \mathrm{H} \mathrm{NMR}\left(\mathrm{CDCl}_{3}\right)$

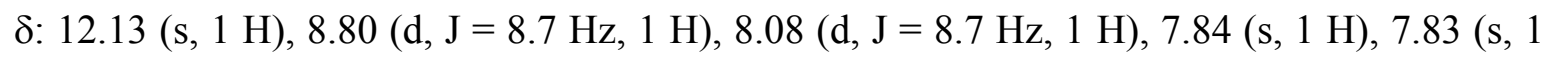
H), $7.60\left(\mathrm{q}, \mathrm{J}_{1}=7.2 \mathrm{~Hz}, \mathrm{~J}_{2}=14.7 \mathrm{~Hz}, 1 \mathrm{H}\right), 7.17(\mathrm{t}, \mathrm{J}=7.8 \mathrm{~Hz}, 1 \mathrm{H}), 4.32-4.25(\mathrm{~m}, 6 \mathrm{H})$, 1.91-1.29 (m, $24 \mathrm{H}), 0.91-0.81(\mathrm{~m}, 9 \mathrm{H})$. MS (EI): $\mathrm{m} / z 659\left(\mathrm{M}^{+}\right)$. Anal. Calcd. for $\mathrm{C}_{33} \mathrm{H}_{47} \mathrm{NO}_{7}$ : C, 69.60; H, 8.26; N, 2.46. Found: C, 69.95; H, 8.44; N, 2.37.

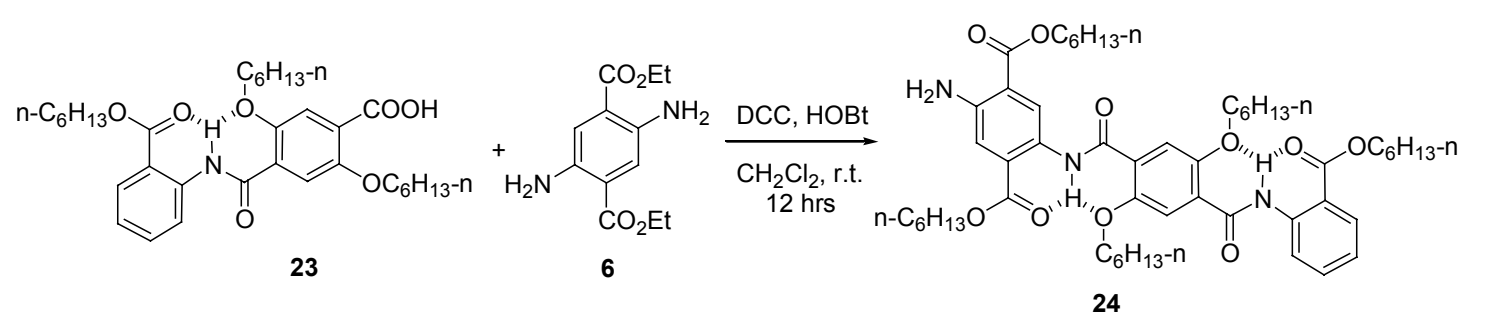

Compound 24. A solution of 23 (0.15 g, $0.26 \mathrm{mmol}), 6(0.066 \mathrm{~g}, 0.26 \mathrm{mmol})$, DCC (0.054 g) and $\mathrm{HOBT}(0.036 \mathrm{~g})$ in $\mathrm{CH}_{2} \mathrm{Cl}_{2}(12 \mathrm{~mL})$ was stirred at room temperature for 12 hours and then concentrated under reduced pressure. The crude product was purified by column chromatography $\left(\mathrm{CH}_{2} \mathrm{Cl}_{2}\right)$ to obtain the desired product $(0.12 \mathrm{~g}, 56 \%)$ as a yellow solid. M.p. 85-87 ${ }^{\circ} \mathrm{C} .{ }^{1} \mathrm{H}$ NMR $\left(\mathrm{CDCl}_{3}\right): \delta 12.10(\mathrm{~s}, 1 \mathrm{H}), 11.54$ (s, $\left.1 \mathrm{H}\right), 9.17$ (s, $\left.1 \mathrm{H}\right), 8.08(\mathrm{~s}, 1 \mathrm{H}), 8.05$ (s, 1 H), 7.59-7.58 (m, 1 H), 7.38 (d, J = 2.7 Hz, 1 H), 7.15 (t, J = 7.2 Hz, 1 H), 4.43-4.25 (m, $10 \mathrm{H}), 1.88-1.73$ (m, $6 \mathrm{H}), 1.50-1.36(\mathrm{~m}, 27 \mathrm{H}), 0.93-0.83$ (m, 9 H). MS (MALDI): $m / z$ 579.3 $\left(\mathrm{M}+\mathrm{Na}-\mathrm{C}_{14} \mathrm{H}_{18} \mathrm{NO}_{3}\right)^{+}$. Anal. Calcd. for $\mathrm{C}_{45} \mathrm{H}_{61} \mathrm{~N}_{3} \mathrm{O}_{10}: \mathrm{C}, 67.25 ; \mathrm{H}, 7.60 ; \mathrm{N}, 5.23$. Found: $\mathrm{C}$, $67.49 ; \mathrm{H}, 7.81 ; \mathrm{N}, 5.14$.

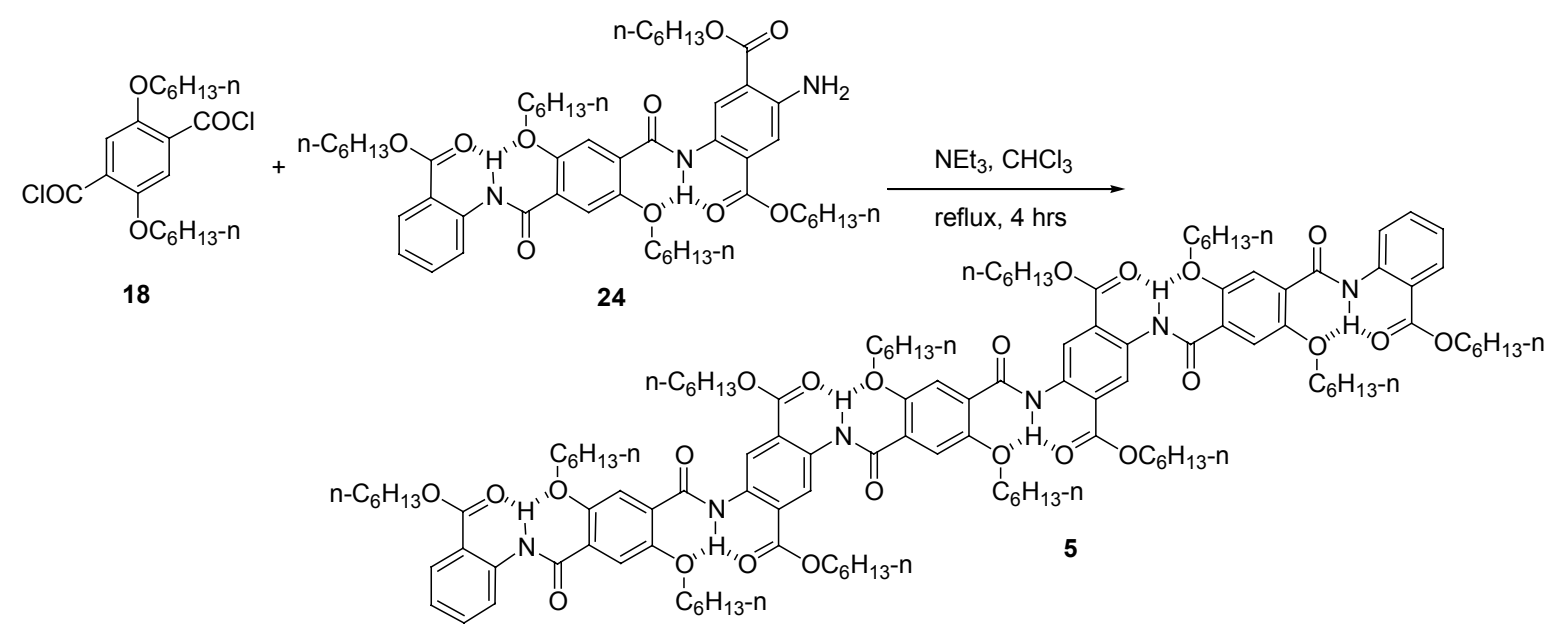

Compound 5. Diacid $17(28.0 \mathrm{mg}, 0.077 \mathrm{mmol})$ was first refluxed in thionyl chloride $(2 \mathrm{~mL})$ 
to give 18, which was used directly for the next step. Treatment of the above 18 with 24 (140 $\mathrm{mg}, 0.155 \mathrm{mmol})$ in refluxed chloroform $(10 \mathrm{~mL})$ in the presence of $\mathrm{NEt}_{3}(0.2 \mathrm{~mL})$ for 4 hours afforded 5 (112 $\mathrm{mg}, 85 \%)$ as a pale green solid after work-up and column chromatography $\left(\mathrm{CH}_{2} \mathrm{Cl}_{2}\right)$. M.p. $230-232{ }^{\circ} \mathrm{C} .{ }^{1} \mathrm{H} \mathrm{NMR}\left(\mathrm{CDCl}_{3}\right): \delta 12.12$ (s, $\left.3 \mathrm{H}\right), 12.06$ (s, 3 H), 9.52 (s, 4 H), 8.82 (d, J = 8.1 Hz, 2 H), 8.07 (d, J = 6.6 Hz, 2 H), 7.87-7.82 (m, 6 H), 7.60 (t, J = 7.2 Hz, $2 \mathrm{H}), 7.16(\mathrm{t}, \mathrm{J}=6.9 \mathrm{~Hz}, 2 \mathrm{H}), 4.47-4.26(\mathrm{~m}, 24 \mathrm{H}), 1.91-1.75(\mathrm{~m}, 16 \mathrm{H})$, 1.48-1.21 (m, 48 H), 0.93-0.83 (m, $24 \mathrm{H})$. MS (MALDI): $m / z 1960.9(\mathrm{M}+\mathrm{Na})^{+}$. Anal. Calcd. for $\mathrm{C}_{110} \mathrm{H}_{148} \mathrm{~N}_{6} \mathrm{O}_{24}: \mathrm{C}, 68.16 ; \mathrm{H}, 7.70 ; \mathrm{N}, 4.34$. Found: 68.66; H, 7.93; N, 4.18. 
Packing diagram of compound 2:

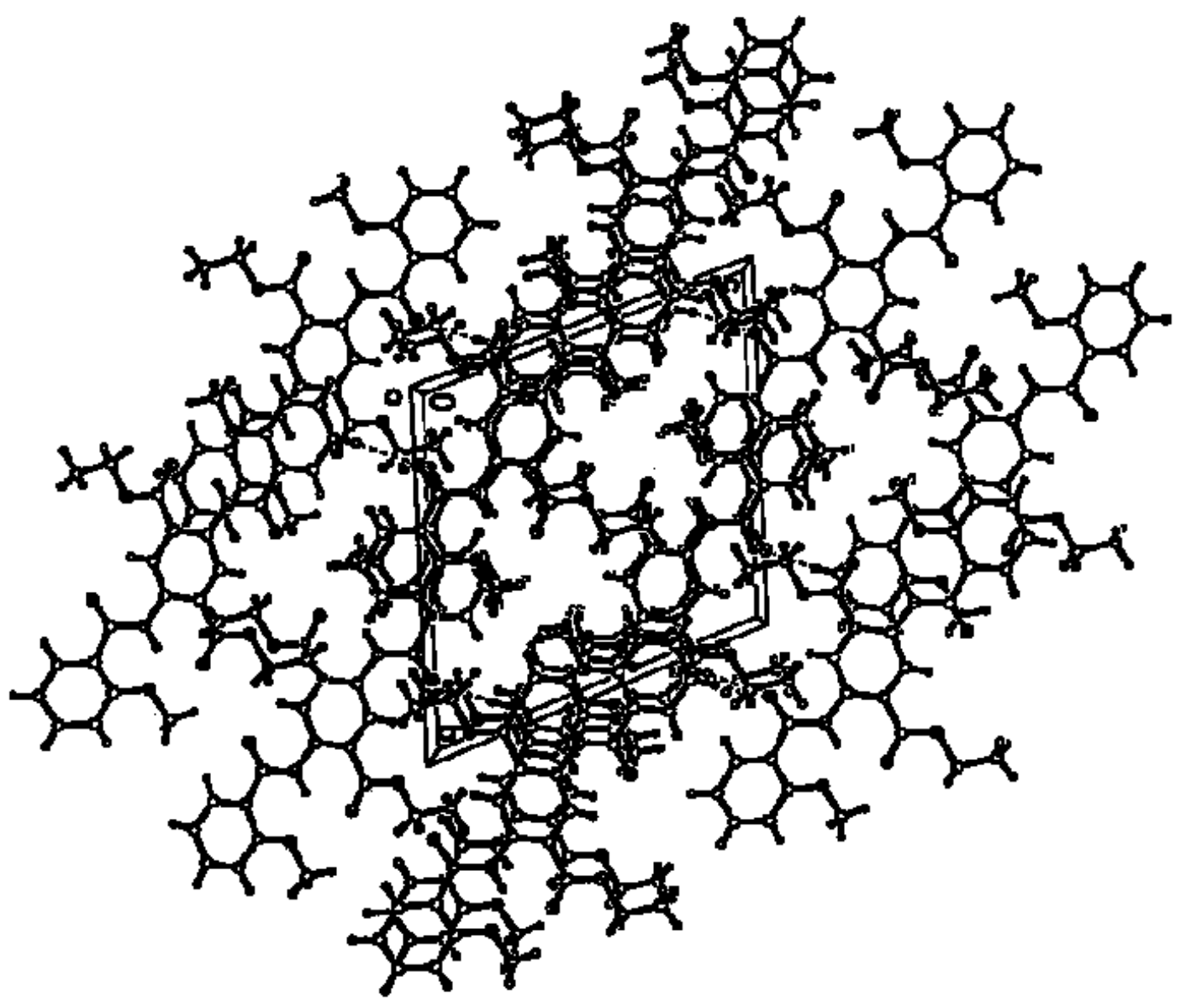


Packing diagram of compound 3:

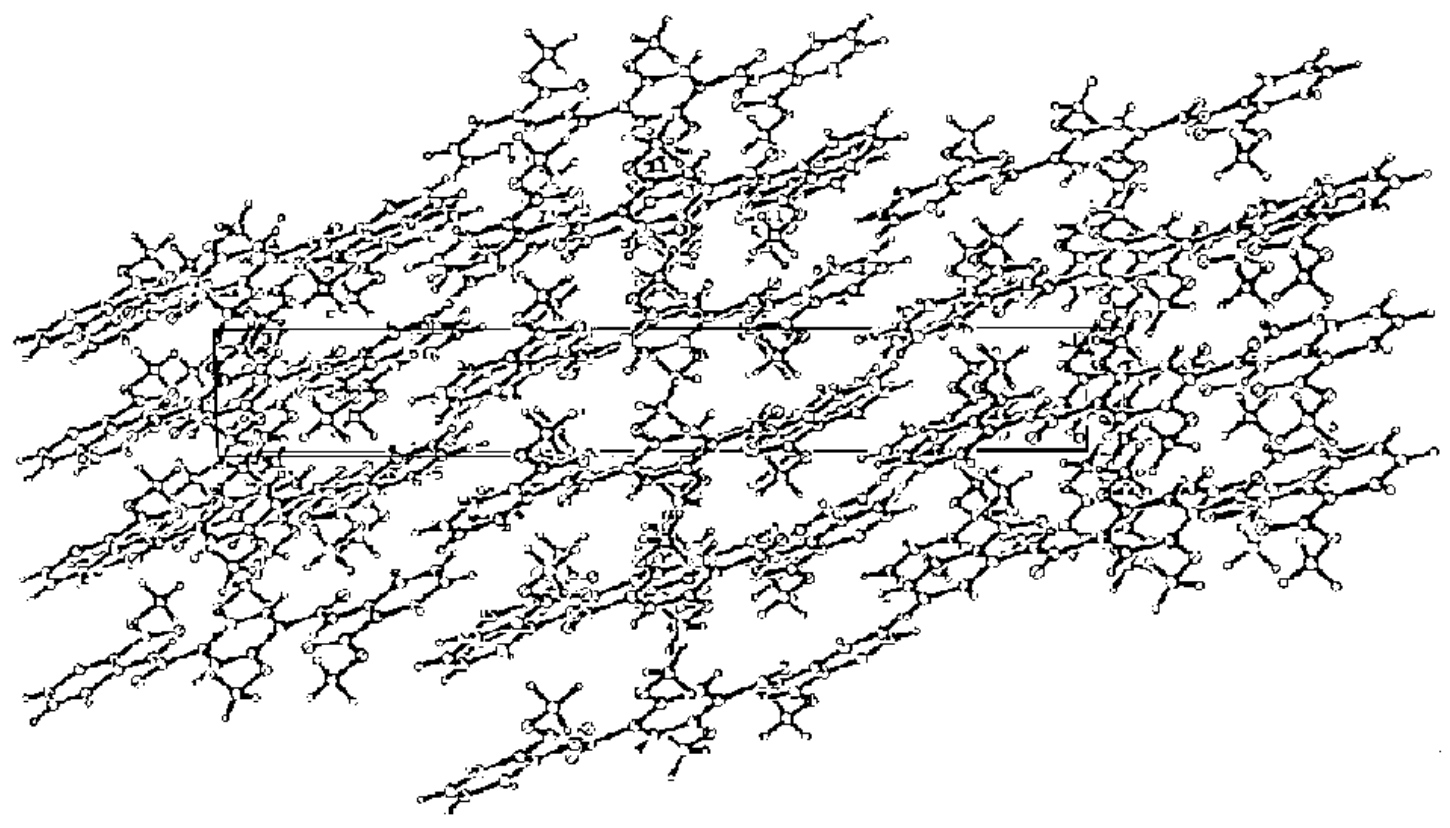

